\title{
Protesta social y estadios del desarrollo moral: una propuesta analítica para el estudio de la movilización social del siglo XXI
}

\section{Santiago Giraldo-Luque ${ }^{1}$}

Recibido: 2017-06-14

Enviado a pares: 2017-06-16
Aprobado por pares: 2017-07-12

Aceptado: 2017-08-15

DOI: 10.5294/pacla.2018.21.2.9

Para citar este artículo / to reference this article / para citar este artigo Giraldo-Luque, S. (2018). Protesta social y estadios del desarrollo moral: una propuesta analítica para el estudio de la movilización social del siglo XXI. Palabra Clave, 21(2), 469-498. DOI: $10.5294 /$ pacla.2018.21.2.9

\section{Resumen}

Diferentes estudios sobre la democracia y la desafección política han considerado que internet y las redes sociales otorgan nuevas oportunidades para la movilización social y la participación ciudadana. Las movilizaciones sociales de la segunda década del siglo XXI, como la Primavera Árabe o el $15 \mathrm{M}$ en España, definieron el protagonismo de la comunicación en línea y de las redes sociales para la convocatoria y el desarrollo de acciones de protesta. El artículo, que utiliza los estadios del desarrollo moral y los aplica a la realidad social, aporta una propuesta teórica de análisis tridimensional -introduce los conceptos de estrategia, acción y objetivo - para el estudio de las movilizaciones sociales contemporáneas. Además, expone y compara las tres dimensiones analíticas y las utiliza para caracterizar de forma exploratoria tres casos de movilización social (la Primavera Árabe egipcia, el

1 orcid.org/0000-0003-0024-7081. Universidad Autónoma de Barcelona, España. santiago.giraldo@uab.cat 
15M y la Plataforma de Afectados de la Hipoteca en España). Dentro del texto, el uso de las redes sociales y de los medios de comunicación es un elemento de estudio transversal que se sitúa sobre las tres dimensiones propuestas. El análisis realizado demuestra que algunas expresiones de protesta han decaído hacia sentimientos de frustración política ciudadana, han sido desplazadas a un segundo plano institucional o han sufrido una sistemática desarticulación. El estudio propone la conceptualización del movimiento social contemporáneo como un espacio colectivo construido sobre una estrategia dirigida a un sistema político concreto, que realiza acciones de cambio centradas en procesos de autonomía y que se traza objetivos consensuados de largo plazo.

\section{Palabras clave}

Movimientos sociales; participación; redes sociales; democracia; ciudadanía; emoción; estadio moral (Fuente: Tesauro de la Unesco). 


\section{Social Protest and Stages of Moral Development: An Analytical Proposal for the Study of Social Mobilization in the $21^{\text {st }}$ Century}

\section{Abstract}

Different studies on democracy and political disaffection are of the belief that the internet and social networks provide new opportunities for social mobilization and citizen participation. The social mobilizations of the second decade of the $21^{\text {st }}$ century, such as the Arab Spring or the 15M in Spain, defined the protagonist role of online communication and social networks for the summon and the development of protests. This paper, which uses the stages of moral development and applies them to social reality, provides a theoretical proposal for a three-dimensional analysis (introducing the concepts of strategy, action, and objective) to study contemporary social mobilizations. It also presents and compares the three analytical dimensions and uses them to characterize, in an exploratory manner, three cases of social mobilization (the Arab Spring in Egypt, the 15M, and the Platform for People Affected by Mortgages in Spain). In the text, the use of social networks and the media is a transverse study based on the three dimensions proposed. The analysis shows that some forms of protest have declined to the feelings of political frustration of citizens, and they have been displaced to a second institutional level or suffered a systematic disarticulation. We propose the conceptualization of the contemporary social movement as a collective space built on a strategy aimed at a specific political system that performs change actions focused on autonomy processes and sets out long-term objectives that have been agreed upon.

\section{Keywords}

Social movements; participation; social networks; democracy; citizenship; emotion; moral stage (Source: Unesco Thesaurus). 


\section{Protesta social e estágios do desenvolvimento moral: uma proposta analítica para 0 estudo da mobilização social do século XXI}

\section{Resumo}

Diferentes estudos sobre a democracia e a desafeição política têm considerado que a internet e as redes sociais outorgam novas oportunidades para a mobilização social e a participação cidadã. As mobilizações sociais da segunda década do século XXI, como a Primavera Árabe ou o 15M na Espanha, definiram o protagonismo da comunicação online e das redes sociais para a convocatória e o desenvolvimento de ações de protesto. Este artigo, que utiliza os estágios do desenvolvimento moral e os aplica à realidade social, proporciona uma proposta teórica de análise tridimensional —introduz os conceitos de estratégia, ação e objetivo - para o estudo das mobilizações sociais contemporâneas. Além do mais, expõe e compara as três dimensões analíticas e as utiliza para caracterizar de forma exploratória três casos de mobilização social (a Primavera Árabe egípcia, o $15 \mathrm{M}$ e a Plataforma de Afetados pela Hipoteca na Espanha). No texto, o uso das redes sociais e dos meios de comunicação é um elemento de estudo transversal situado sobre as três dimensões propostas. A análise realizada demonstra que algumas expressões de protesto transformaram-se em sentimento de frustração política cidadã, passaram a um segundo plano institucional ou sofreram uma sistemática desarticulação. Propõe-se a conceptualização do movimento social contemporâneo como um espaço coletivo construído sobre uma estratégia dirigida a um sistema político concreto, que realiza ações de mudanças focadas em processos de autonomia e que traça objetivos consensuais em longo prazo.

\section{Palavras-chave}

movimentos sociais; participação; redes sociais; democracia; cidadania; emoção; estágio moral (Fonte: Tesauro da Unesco). 


\section{Introducción}

A partir de la crisis o burbuja puntocom a finales del siglo $\mathrm{XX}$ y principios del XXI, los discursos utópicos seguidos de la introducción masiva de una nueva tecnología de comunicación —en este caso las redes sociales-como motor del cambio económico y como espacio de apertura social y política (McLuhan, 1969; Wiener, 1969; Bennett y Segerberg, 2012; Castells, 2012) iniciaron un proceso de transformación crítica y analítica (Žižek, 2010; Van Dijk, 2011; Fuchs, 2014; Ceron y Memoli, 2016). Sin duda, interesante en un momento histórico en el que los nuevos medios de comunicación y sus dispositivos electrónicos se han multiplicado y se han convertido en una extensión más del cuerpo humano (Žižek, 2010).

La discusión sobre las posibilidades del fortalecimiento y la participación a partir del uso de internet ha alcanzado dos grandes líneas de discusión con sus respectivos posicionamientos (Giraldo-Luque, 2012, p. 21). Una primera línea identifica internet como un espacio de superación de estructuras representativas y aboga por una versión directa de la democracia —ligada a posturas de corte más utopistas (Castells, 2012; Natale y Ballatore, 2014) - , mientras que otros discursos aprecian las nuevas posibilidades de comunicación como una oportunidad para mejorar los canales representativos (Chadwick, 2006, Anduiza, Cristancho y Sabucedo, 2014; Tufte, 2015; Salzman, 2016).

La segunda línea de debate presenta la discusión clásica entre los ciberoptimistas (Lévy, 2002; Eltantawy y Wiest, 2011) y los ciberpesimistas (Žižek, 2010; Fuchs, 2014; Sunstein, 2017) con posturas intermedias que reflejan virtudes y defectos de internet como herramienta y no como fin de la participación política (Habermas, 2005; Chadwick, 2006; Anduiza, Cristancho y Sabucedo, 2014; Tufte, 2015).

A partir de las dos discusiones anteriores, las nuevas formas de organización de la protesta social mediadas por las redes sociales (Ortiz-Galindo, 2016a) han sido también objeto de análisis. Por una parte, se ha estudiado el contagio emocional producido en una red social y que puede conducir a una movilización física. Los análisis se han centrado en experimentos de 
manipulación de los mensajes que los usuarios reciben en sus redes sociales (Bond et al., 2012; Coviello et al., 2014; Jones, Bond, Bakshy y Fowler, 2017). Por otra parte, se han establecido diferencias entre la protesta tradicional y las movilizaciones que implican un alto uso de las redes sociales (Anduiza, Cristancho y Sabucedo, 2014), con lo que se ha consolidado el concepto de connective action (Bennett y Segerberg, 2012). En este se ponen de manifiesto dos características propiciadas por el uso de la comunicación digital y las redes sociales. En primer lugar, el contenido político toma la forma de marcos generales que pueden ser personalizados y adaptados a diferentes contextos (Anduiza, Cristancho y Sabucedo, 2014, p. 753). Proceso que implica la pérdida de la centralidad política institucional (Bentivegna, 2006, p. 332) sin que exista la necesidad de una adhesión o compromiso individual o grupal a una organización ideológica previa (Cammaerts y Van Audenhove, 2005).

La segunda característica remarca la posibilidad de que los ciudadanos compartan recursos cognitivos a través de redes sociales de confianza y alejen la necesidad de recurrir a instituciones tradicionales. Anduiza, Cristancho y Sabucedo (2014, p. 753), Eltantawy y Wiest (2011) y Castells (2012) sitúan también una alta expectación y un protagonismo sobre las redes sociales, pues pueden garantizar un proceso de movilización y asegurar un papel activo y participativo de la ciudadanía en una acción colectiva.

La recopilación realizada por Ortiz-Galindo (2016a, p. 165) describe, asimismo, cómo los estudios sobre la nueva ola de cibermovimientos sociales se han referido principalmente a las transformaciones de recursos comunicativos y de los repertorios de acción (donde bien puede ubicarse este trabajo), a la aparición de un nuevo tipo de movimientos en la era de las redes y a las revisiones y organizaciones sobre el marco teórico relativo a la movilización social.

A pesar de que los estudios catalogan las redes sociales e internet como escenarios imprescindibles del cambio político, y como herramientas que facilitan la participación (Castells, 2012), el resultado real de las acciones de protesta no es evidente (Torrego y Gutiérrez, 2016) y, en la 
mayoría de los casos, los ejemplos de protestas ciudadanas se han extinguido, bien porque los sistemas políticos han reaccionado al desafío de la protesta (Lynch, Freelon y Aday, 2017), o bien porque las mismas acciones ciudadanas se han reconvertido en movimientos políticos institucionalizados (Casero-Ripollés, Feenstra y Tormey, 2016). La clave del éxito de la movilización social que emergió entre 2011-2012 consistía en el mantenimiento de la protesta establecida a través de la asociación y de la construcción de lazos. Un requisito que significaba la articulación de una base popular amplia y activa bajo un entendimiento común (Chomsky, 2011, p. 11), una condición que fue imposible salvo en grupos o comunidades organizadas más pequeñas y que tenían un trabajo anterior acumulado. Esto empieza a perfilar una crítica a discursos, como el de Cammaerts y Van Audenhove (2005), cuando señalan como característica de las nuevas movilizaciones una configuración a partir del uso de internet, aun sin tener una vinculación previa a una organización colectiva que teja una identidad (Tufte, 2015).

Este artículo propone un marco de análisis para el estudio de las movilizaciones sociales contemporáneas y cambiantes (Ortiz-Galindo, 2016a), que se identifican por destinar una parte importante de sus recursos a la comunicación mediada por las redes sociales (Feenstra, Tormey, CaseroRipollés y Keane, 2016). El texto parte de los estadios del desarrollo moral aplicados al universo social (Habermas, 1981; Alútiz, 2004; Martínez, 2011) e introduce tres conceptos —estrategias, acciones y objetivos - que marcan las dimensiones del análisis que se aplica para definir el estadio de desarrollo de cada una de las movilizaciones estudiadas. El artículo emplea de forma exploratoria las dimensiones teóricas analizadas a tres movilizaciones específicas, realiza una propuesta de clasificación de ellas en función de su estadio de desarrollo y se suma a las críticas de la participación centrada en las redes sociales e internet. A partir de la aplicación del modelo analítico, el texto critica la ausencia de estrategias en algunos de los casos estudiados, la centralidad de las acciones sobre el manejo, la gestión y la búsqueda de trending topics en redes sociales como Twitter o Facebook y la escasa permanencia temporal de las movilizaciones en función de la ausencia de una identidad colectiva. 


\section{Metodología: presentación del marco analítico}

El marco analítico presentado está construido, en primer lugar, a partir de la teoría de los estadios del desarrollo moral propuesta inicialmente por Kohlberg (1992). El autor, quien retoma una primera aproximación de los estadios morales de Piaget (1977), es complementado posteriormente por Habermas (1981), quien acerca la teoría de los estadios a la comprensión de procesos históricos o sociales (Martínez, 2011). Los estadios del desarrollo moral aplicados a fenómenos sociales han sido utilizados por Alútiz (2004) para presentar una explicación histórica y sociológica de las comunidades humanas y por Martínez (2011, p. 27), quien retoma la propuesta habermasiana y propone categorías básicas de relación entre los tipos de acción, las estructuras cognitivas y las perspectivas sociales. Martínez (2011) también señala el aporte del tránsito de Kohlberg a Habermas al afirmar que "el trabajo habermasiano hace compatibles y coherentes una teoría del desarrollo moral con una teoría general de la sociedad” (p. 12).

El análisis propuesto retoma los estadios de desarrollo moral — preconvencional, convencional y posconvencional-y los relaciona con las actuaciones y los comportamientos de las movilizaciones sociales a partir de tres dimensiones de estudio: estrategias, acciones y objetivos de cada uno de los casos planteados. La tabla 1 realiza una adaptación de los rasgos básicos de cada uno de los estadios del desarrollo moral aplicables a la movilización social.

El análisis relacional entre los estadios de desarrollo moral presentados y las dimensiones de estrategia, acción y objetivos requieren la conceptualización de las dimensiones y de la asignación de características para cada una de ellas en función de los tres estadios de desarrollo. Así, el estudio entiende por la estrategia de una movilización social la correspondencia entre las acciones elegidas como expresión de la protesta o manifestación (marchas, ocupaciones, huelgas, uso de la violencia, etc.) y la evolución democrática del sistema político en el que se desarrolla la acción. En este caso, los marcos generales de acción, que pueden ser personalizados o adaptados (Anduiza, Cristancho y Sabucedo, 2104; Castells, 2012) pueden ser analizados en función de la adopción de estrategias de 


\section{Tabla 1. Marco general de análisis: características básicas de los estadios de desarrollo moral}

\begin{tabular}{|c|c|c|c|}
\hline & Características básicas & Niveles de universo simbólico & $\begin{array}{l}\text { Perspectivas sociales e idea de } \\
\text { la justicia }\end{array}$ \\
\hline בี & $\begin{array}{l}\text { Modelo de comportamiento particular, } \\
\text { individual } \\
\text { Normas y expectativas sociales externas } \\
\text { Interés situado en la aprobación social } \\
\text { Interacción autoritaria } \\
\text { Acción individualista e instrumental }\end{array}$ & $\begin{array}{l}\text { Orientación guiada por la acción } \\
\text { Nula inserción en el mundo } \\
\text { simbólico } \\
\text { Agentes a los que se pueden } \\
\text { adscribir intenciones } \\
\text { comprensibles, pero no sujetos con } \\
\text { acciones imputables }\end{array}$ & $\begin{array}{l}\text { Autoridad guiada por personas } \\
\text { de referencia } \\
\text { Orientación hacia la } \\
\text { recompensa y el castigo }\end{array}$ \\
\hline 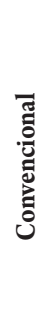 & $\begin{array}{l}\text { Comportamiento generalizado } \\
\text { socialmente } \\
\text { Acción funcional y sistémica } \\
\text { Interacción orientada por las normas } \\
\text { sociales } \\
\text { Reglas como expectativas o acuerdos de } \\
\text { la sociedad }\end{array}$ & $\begin{array}{l}\text { Satisfacción de necesidades a } \\
\text { partir de expectativas socialmente } \\
\text { reconocidas } \\
\text { Expectativas generalizadas de } \\
\text { comportamiento }\end{array}$ & $\begin{array}{l}\text { Autoridades interiorizadas } \\
\text { hacia la lealtad (individual) y la } \\
\text { legalidad (impersonal) } \\
\text { Identificación como miembro } \\
\text { de la sociedad: normas y } \\
\text { contrato social }\end{array}$ \\
\hline 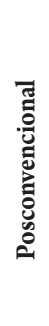 & $\begin{array}{l}\text { Perspectiva de principios } \\
\text { Los principios morales prevalecen sobre } \\
\text { las reglas } \\
\text { Acción discursiva/comunicativa } \\
\text { Definición de valores y de principios } \\
\text { autoescogidos }\end{array}$ & $\begin{array}{l}\text { Proceso de interpretación de las } \\
\text { necesidades } \\
\text { Formación discursiva de la voluntad } \\
\text { que orienta la acción y la crítica } \\
\text { Identificación de contextos vitales } \\
\text { objetivos }\end{array}$ & $\begin{array}{l}\text { Comprensión y aplicación de } \\
\text { normas reflexivas } \\
\text { Interés situado en el bienestar } \\
\text { (social) } \\
\text { Orientación a los principios de } \\
\text { la justicia y a los procedimientos } \\
\text { de fundamentación de las } \\
\text { normas }\end{array}$ \\
\hline
\end{tabular}

Fuente: Kohlberg (1992, pp. 187-195), Habermas

(1992, pp. 61-80), Alútiz (2004, pp. 18-39) y Martínez (2011, p. 27).

una determinada movilización social: no es lo mismo realizar una marcha en El Cairo que en Madrid o Barcelona. Al mismo tiempo, la estrategia describe el marco emocional o racional del contagio o masificación de la protesta, a partir del planteamiento de Bond et al. (2012), Coviello et al. (2014) y Jones et al. (2017). Una acción de protesta que no entiende y apropia su contexto - y sus propias posibilidades de generar desafíos al sistema político - será incorporada a la cotidianidad y propiciará tanto un refuerzo institucional como un aumento de su legitimidad dada su capacidad para gestionar una crisis. 
La segunda dimensión, la acción, se introduce como el tipo de metas que se plantean por el colectivo y como la capacidad de la protesta para alcanzar sus metas sin requerir o exigir una respuesta institucional. Las propuestas de Bentivegna (2006), Anduiza, Cristancho y Sabucedo (2014) y Ortiz-Galindo (2016a) quedan reflejadas al intentar desligar la acción de movilización de una centralidad institucional. Asimismo, el tipo de respuesta de la institución — sistémica o no- permite también perfilar el alcance de las metas definidas por la movilización. Un último aspecto por considerar es introducido por Castells (1997) al mencionar el concepto de intencionalidad de la acción, recogido por Ortiz-Galindo (2016a) en su caracterización del "cibermovimiento social".

Por último, los objetivos de la movilización social se asumen como la proyección de las acciones de protesta a corto, medio y largo plazo, así como la identificación de la lucha social con una temática asociada a los derechos humanos (salud, educación, vivienda y justicia), lo cual establece una línea de actuación coherente, diversificada, autónoma, dinámica y representada en actuaciones concretas (Habermas, 1981). En esta dimensión, es relevante la discusión de Cammaerts y Van Audenhove (2005) cuando introducen la poca adscripción a organizaciones previas de los nuevos movimientos sociales, y el planteamiento de Bond et al. (2012) y Jones et al. (2017) al analizar el contagio emotivo en la estructuración de un evento de protesta que requiere una proyección articulada de organización social en el tiempo. De igual forma, los objetivos apropian la concepción de Touraine (2005) sobre la identidad colectiva necesaria para la construcción de un movimiento social y que le permite a la acción colectiva tener una continuidad temporal (Larraña, 1999; Ortiz-Galindo, 2016a).

La propuesta de análisis se complementa con la caracterización relacional de los estadios de desarrollo moral con las tres dimensiones introducidas. La vinculación se realiza en función de los rasgos que definen el comportamiento de la movilización analizada y, dentro de ellos, se escenifica el papel de los medios y de las redes sociales a partir de su articulación dentro de la movilización como estrategia, acción u objetivo. 


\section{Estadio preconvencional}

\section{Estrategia}

Ausencia de estrategia y análisis estrecho del sistema político: los marcos generales (Anduiza, Cristancho y Sabucedo, 2014) no se ajustan a las condiciones del sistema político concreto. La acción no genera desafíos al sistema político y la movilización es asimilada institucionalmente (Martínez, 2011). En su respuesta efectiva, el sistema gana legitimidad. Estrategia delineada por vínculos emocionales básicos: miedo, rabia e ira, canalizados a través de las redes sociales (Bond et al., 2012; Coviello et al., 2014; Jones et al., 2017). Alta expectación puesta en las redes sociales (Anduiza, Cristancho y Sabucedo, 2014) como motor del cambio. Aumento de la tensión social al crecer la polarización en el discurso mediático (Lynch, Freelon y Aday, 2017).

\section{Acción}

La intencionalidad de la acción (Castells, 1997) está desdibujada y mediada por lo concreto e inmediato, al tiempo que está caracterizada por la contundencia o la violencia. La acción no pierde la centralidad institucional (Bentivegna, 2006; Anduiza, Cristancho y Sabucedo, 2014; Ortiz-Galindo, 2016a) al exigir una respuesta a la misma institución. La institución responde a la demanda de cambio, solo si no consigue diezmarla por la coacción o si se convierte en una amenaza sistémica. Las promesas o cambios ofertados institucionalmente merman las protestas que se diluyen por el uso (o la amenaza de uso) de la violencia (Martínez, 2011). Los medios y las redes sociales son utilizadas como mecanismos de presión sobre el marco institucional (Eltantawy y Wiest, 2011), aunque existen intentos claros para establecer marcos de censura y control informativo.

\section{Objetivo}

Objetivos de corto plazo (Larraña, 1999), sin continuidad. No existe una identidad colectiva identificada con un derecho insatisfecho (Touraine, 2005). La demanda de derechos se realiza sin consenso previo (Habermas, 1981) y no se trazan acciones para construir o gestionar el derecho de forma autónoma (Cammaerts y Van Audenhove, 2005). Los objetivos están centrados en una única acción, fácilmente asimilable. Las redes sociales se utilizan 
con un objetivo único de denuncia, pero pierden rápidamente su capacidad de impacto (Fuchs, 2014). La movilización en la red sigue la lógica del trending topic y no se desarrollan intercambios de recursos cognitivos a través de redes sociales de confianza (Anduiza, Cristancho y Sabucedo, 2014).

\section{Estadio convencional}

\section{Estrategia}

Análisis del sistema político que define la acción dentro de un marco de actuación concreto: las normas sociales (Martínez, 2011). Las acciones se personalizan en función del contexto, pero siempre están ligadas al marco institucional (Anduiza, Cristancho y Sabucedo, 2014). Ausencia de desafíos sistémicos, porque el movimiento, autorregulado, se adecúa a los espacios reglados propios de los escenarios de protesta institucionalizados (Habermas, 1981). Estrategia delineada por un vínculo de emociones sofisticado: esperanza e indignación (Bond et al., 2012; Coviello et al., 2014; Jones et al., 2017). Alta expectación puesta en las redes sociales (Anduiza, Cristancho y Sabucedo, 2014) como plataformas de trasformación.

\section{Acción}

La intencionalidad de la acción se define por lo inmediato (Castells, 1997). Las metas están ligadas al posicionamiento de agendas y actores dentro del escenario institucional (Ortiz-Galindo, 2016a). El uso de medios y redes sociales es una de las acciones de movilización y se convierte en su marco de descentralidad institucional (Feenstra et al., 2016). La red social se convierte, por momentos, en la movilización. Las acciones se centran en el impacto mediático, trending topics, y en tener un alcance alto de interacción (Giraldo-Luque, 2015). La movilización exige y recibe respuestas institucionales convencionales y no violentas (Bentivegna, 2006). La movilización es integrada al sistema institucional y, en algunas ocasiones, al sistema de reparto de poder tradicional (Casero-Ripollés, Feenstra y Tormey, 2016).

\section{Objetivo}

Objetivos de corto plazo centrados en el impacto sobre la agenda política temática (Larraña, 1999). Inicio de un proceso de construcción de identidad sobre algunos asuntos de protesta (Touraine, 2005). Objetivos de 
medio plazo si el movimiento es institucionalizado y participa en el juego de poder (elecciones) e intenta el cambio desde una perspectiva sistémica (Casero-Ripollés, Feenstra y Tormey, 2016). Pocos consensos previos sobre derechos fundamentales, ausencia de acciones orientadas a su construcción (Cammaerts y Van Audenhove, 2005). Algunas acciones diversas y creativas, con diferentes resultados, siempre bajo un marco legal (Habermas, 1981) y bajo el intercambio de algunos recursos cognitivos en redes sociales de confianza (Anduiza, Cristancho y Sabucedo, 2014).

\section{Estadio posconvencional}

\section{Estrategia}

Análisis estratégico del sistema político (Anduiza, Cristancho y Sabucedo, 2014) que personaliza, adapta y crea acciones orientadas a proponer desafíos al sistema político. Primacía de una perspectiva moral identificada con principios de justicia que marca un escenario discursivo antecesor a la acción (Habermas, 1981). Estrategia delineada por vínculos racionales decididos colectivamente y vinculados a su identidad (Touraine, 2005). El actor se define desde una perspectiva racional-consensual y utiliza la emoción (Bond et al., 2012; Coviello et al., 2014; Jones et al., 2017) con el objetivo de generar legitimidad social hacia sus acciones. Las redes sociales desempeñan un papel estratégico respecto de la convocatoria y la comunicación de sus acciones (Alonso-Muñoz y Casero-Ripollés, 2016). Baja expectación puesta en las redes sociales, integradas en una estrategia racional de comunicación digital (Anduiza, Cristancho y Sabucedo, 2014), con una unidad discursiva clara (Lynch, Freelon y Aday, 2017).

\section{Acción}

Clara intencionalidad de las acciones (Castells, 1997) definida hacia la satisfacción de derechos. Consensos sobre las acciones (Alútiz, 2010). La movilización provoca impulsos jurídicos/políticos y promueve arreglos que buscan adecuar/integrar las demandas (Carmona, 2017). El movimiento identifica vacíos normativos y justificaciones morales que blindan la lucha social. La acción se desmarca de la institucionalidad (Bentivegna, 2006; Anduiza, Cristancho y Sabucedo, 2014; Ortiz-Galindo, 2016a) y la obliga a reaccionar por fuera de su sistema reglado. El derecho puede ejer- 
cerse de forma autónoma sin que medie la intervención institucional (Giraldo-Luque, 2015).

Sus acciones no requieren respuestas institucionales para alcanzar sus objetivos. Medios y redes sociales como factor de presión adicional: acompañan las acciones que realiza el movimiento para lograr legitimidad social. Los medios forman parte de acciones estratégicas, como herramientas (Feenstra et al., 2016).

El marco general de análisis descrito fue aplicado sobre tres casos seleccionados intencionadamente (tabla 2) de acuerdo con su importancia mediática y su adaptación a los modelos analíticos presentados con una intención metodológica exploratoria y explicativa. El método implementado aplica las dimensiones de análisis a los casos y compara las apreciaciones con aproximaciones que dialogan con el modelo. Cada caso es caracterizado y analizado en función de su definición de estrategias, acciones y objetivos, con la intención de clasificar diferentes tipos o estadios de movilización social.

Tabla 2. Relación de la muestra de estudio

\begin{tabular}{|c|c|c|}
\hline Acción de protesta & País & Periodo de vigencia de acciones \\
\hline Revolución egipcia & Egipto & Enero de 2011-julio de 2014 \\
\hline $15 \mathrm{M}$ & España & Mayo-julio de 2011 \\
\hline Plataforma de Afectados por la Hipoteca & España & Febrero de 2009-actualidad \\
\hline
\end{tabular}

Fuente: Elaboración propia.

\section{Análisis y resultados}

\section{Un caso preconvencional, la Revolución egipcia}

A pesar de que la Revolución egipcia fue enarbolada como una plataforma renovadora en una sociedad dominada por la exclusión y por el carácter dictatorial del mandato de Mubarak (1981-2011), cuatro años de transiciones políticas bruscas señalan un camino delineado por la inestabilidad política 
y por el desmantelamiento de la protesta por parte de las autoridades a través de la prohibición de la expresión política, el arresto de líderes revolucionarios y el asesinato de más de 3500 personas (Amnesty International, 2015; Naber y Said, 2016; Lynch, Freelon y Aday, 2017).

\section{La estrategia}

El movimiento egipcio no tenía una estrategia definida, partió de un escenario emotivo (Bond et al., 2012) guiado por la rabia y la ira, y generó una serie de expresiones de violencia y presión social que terminaron por obligar a un cambio de gobierno (Lynch, Freelon y Aday, 2017). Los marcos generales de la protesta (Anduiza, Cristancho y Sabucedo, 2014), en el caso egipcio, se establecen dentro de una estructura cerrada de participación que limita la construcción de alternativas. El marco preconvencional de la protesta no demandó una respuesta sofisticada por parte de las instituciones y no generó un desafío sistémico sobre el marco del reparto del poder egipcio. La respuesta institucional efectiva dada por los militares y el nuevo gobierno en 2014 obtuvo la legitimidad social necesaria para poner fin a tres años de caos (Giraldo-Luque, 2015).

El movimiento o sus exigencias (pan, libertad y justicia) fueron desdibujados por las instituciones que mantuvieron un sistema de decisiones ligado a las élites. El cambio nominal en el poder, a pesar del impacto mediático, sobre todo internacional y en las redes sociales (Eltantawy y Wiest, 2011), no implicó el reparto de derechos para los ciudadanos egipcios (Naber y Said, 2016).

Las respuestas institucionales fueron siempre dadas en el marco de un sistema político con escasas bases democráticas y sin un pacto social legitimado (Habermas, 1981), como quedó demostrado en diferentes intentos de construir una constitución. Las redes sociales, pasada la euforia de la caída de Mubarak, solo causaron un aumento "del conflicto político y facilitaron la propagación del miedo y del odio que terminó por dinamitar la transición política para otorgar todo el apoyo popular al golpe militar" (Lynch, Freelon y Aday, 2017). 


\section{La acción}

En el caso egipcio, la intencionalidad de la acción (Castells, 1997), y de sus metas, estuvo marcada por lo concreto y lo inmediato y se caracterizó por la contundencia y por una constante manifestación de la violencia.

La principal meta del movimiento exigía un cambio en el Gobierno que finalmente se produjo. Sin embargo, a partir de julio de 2013, cuando Mohamed Morsi fue derrocado por las fuerzas armadas, el miedo se apoderó de la población (Castells, 2012) y el movimiento revolucionario disminuyó viendo cómo los militares asumían nuevamente el poder. La vuelta de los militares implicó el desmantelamiento de las voces críticas a través de la coacción física y legal (Amnesty International, 2015; Nadine y Said, 2016).

En Egipto, las demandas tuvieron siempre el foco de la institucionalidad manifiesta, de quien siempre vino una respuesta dentro de las alternativas del sistema político establecido. En ningún caso se vivió una pérdida de la centralidad institucional (Bentivegna, 2006) y, aunque existieron mecanismos como los medios sociales para romper la hegemonía comunicativa y promover espacios de solidaridad social en el mundo (Eltantawy y Wiest, 2011), el marco de censura promovido minó paulatinamente la construcción de posturas críticas al tiempo que disminuían los contagios emotivos promovidos por las redes sociales (Human Right Watch, 2014).

\section{Los objetivos}

Contrario a lo expuesto por Larraña (1999) y Ortiz-Galindo (2016a), los objetivos de la movilización egipcia fueron de corto plazo, no se correspondieron con el resultado de un proceso previo de construcción colectiva (Cammaerts y Van Audenhove, 2005) y estaban centrados en una única meta: cambiar la figura presidencial.

El movimiento de protesta no llegó a consolidar una identidad colectiva (Touraine, 2005) que articulara la protesta y promoviera la autonomía para la satisfacción de derechos incluidos dentro de un marco general de actuación (Anduiza, Cristancho y Sabucedo, 2014). 
Tal ausencia de objetivo evitó, incluso, que líderes políticos cercanos al movimiento alcanzaran el poder a través de la vía electoral, un camino abierto hacia el mundo convencional que no aprovechó la movilización. Al mismo tiempo, dentro de las lógicas de la protesta egipcia, no existió un intercambio de recursos cognitivos que permitieran cohesionar acciones o acercar posturas distantes (Anduiza, Cristancho y Sabucedo, 2014).

Por último, en la Revolución egipcia, las redes sociales convirtieron la primera fase de la protesta en un trending topic mundial y sus acciones inspiraron una serie de movilizaciones sociales en todo el mundo (Eltantawy y Wiest, 2011). Sin embargo, una vez pasada la emoción inicial en la red social, el caso egipcio perdió interés en todos los medios de comunicación. Los episodios de violencia posteriores a la revolución no tuvieron repercusiones en la agenda mediática ni fueron tendencia en las redes.

\section{El 15M, un ejemplo convencional}

El movimiento 15M en España marcó una buena parte de la agenda política y mediática a partir de la primavera de 2011 (Micó y Casero-Ripollés, 2014). Bajo un sentimiento emotivo guiado por la indignación y la esperanza (Castells, 2012), las expresiones de inconformidad social recogidas en el $15 \mathrm{M}$ pueden agruparse como movimientos convencionales en función de que sus actuaciones estuvieron ligadas a un "modelo de comportamiento generalizado socialmente, orientado a una función social y en conformidad con el sistema existente de normas” (Martínez, 2011, p. 27).

\section{La estrategia}

El movimiento que emerge en la primavera de 2011, con gran relevancia mediática y millones de interacciones en redes sociales (Micó y CaseroRipollés, 2014), no representó, en su intencionalidad, un desafío sobre el sistema político-social (Martínez, 2011). Sus acciones, las acampadas, se convirtieron en poco tiempo en convencionales, dejaron de ser sorpresivas, interfirieron poco en la cotidianidad y motivaron una intensificación de los sistemas de control para evitar nuevas protestas similares (Camps y Vergés, 2015). El sistema político, en su marco reglado (Habermas, 2005), respondió, asimiló la amenaza y demostró una capacidad adaptativa que le 
llevó a ganar la legitimidad social que poco a poco fueron perdiendo los indignados. El 15M no planteó un desafío alternativo o autónomo (Touraine, 2005) que propiciara una acción de emergencia de las instituciones, y se estructuró desde su propia autorregulación social y a partir de una interacción reglada convencional (Martínez, 2011).

La institucionalidad respondió desde sus herramientas legales y, cuando lo precisó, ejerció el uso legítimo de la fuerza para responder a algunos episodios violentos (Giraldo-Luque, 2015). En el momento en que se sobrepasaron los límites de lo legal (hacia un marco de protesta preconvencional), los sentimientos de solidaridad con la protesta se acabaron, pues se dejaron de cumplir las expectativas generalizadas de comportamiento (Habermas, 1981; Alútiz, 2004; Martínez, 2011).

A partir de lo señalado por Castells (2012), el 15M se estructuró bajo una perspectiva emocional que se corresponde con una alta expectación sobre los medios sociales (Feenstra, 2016), utilizados como estrategia de contagio emotivo (Bond et al., 2012; Coviello et al., 2014; Jones et al., 2017) sobre marcos generales de convergencia (Anduiza, Cristancho y Sabucedo, 2014).

\section{La acción}

En el plano de la definición de acciones, el 15M buscaba situar en la agenda pública su sentimiento de indignación. No se declara, por tanto, una intencionalidad manifiesta específica (Castells, 1997), pero sí un marco general y amplio - incluso adaptado - de la expresión social colectiva (Anduiza, Cristancho y Sabucedo, 2014). En ese sentido, el papel de las redes sociales, y el eco que tuvieron sus acciones en diferentes ciudades (Casero-Ripollés y Feenstra, 2012), ayudó a cumplir un objetivo básico e inmediato: el mundo conoció el mensaje de indignación transmitido desde España. Pero una vez pasada la emoción, el cansancio y el vacío también tuvieron eco en la red social (Žižek, 2010, p. 158). La indignación, sin precisar una demanda concreta, tuvo por única respuesta la contención referida al orden público, aunque la protesta y sus agendas sí estuvieron vinculadas o dirigidas al marco institucional mediático (Casero-Ripollés y Feenstra, 2012), en contra de lo propuesto por Bentivegna (2006) y Ortiz-Galindo (2016a). 
Dentro de las acciones, resaltar la descentralidad comunicativa lograda por los indignados a partir del uso de los medios y las redes sociales. Desde este ángulo, el marco de trabajo sí se aleja de una posición oficialmediática (Feenstra et al., 2016), aunque se reconoce, por una parte, el impacto de los medios tradicionales en la mediatización y agenciación de los temas relacionados con el movimiento (Micó y Casero-Ripollés, 2014); y por otra, la alta expectativa puesta en las redes sociales para alcanzar un impacto más alto (Anduiza, Cristancho y Sabucedo, 2014).

Uno de los resultados más significativos del 15M, la inclusión de una agrupación política en el Parlamento español, consolida la legitimación del sistema político, pues permite integrar la expresión de protesta dentro de su sistema político-operativo (Giraldo-Luque, 2015). La integración al juego electoral tradicional de una parte de los manifestantes (Casero-Ripollés, Feenstra y Tormey, 2016) es, sin duda, una victoria del convencionalismo sobre un movimiento basado en una emoción sofisticada y construida en conjunto como la indignación (Castells, 2012, p. 31).

\section{Los objetivos}

Los objetivos generales de la indignación, una vez pasado el posicionamiento de la agenda mediática, fueron de corta duración (Larraña, 1999). La dificultad de consensos (Habermas, 2005), la escasa definición de un plan de acción trazado sobre derechos fundamentales u objetivos públicos (OrtizGalindo, 2016a) y la ausencia de acciones específicas que lograran acercar tales derechos a la ciudadanía mermaron rápidamente el factor emocional de la protesta (Castells, 2012) y, salvo algunos colectivos que ya trabajaban a través de una identidad colectiva (Touraine, 2005; Chomsky, 2011), se pasó de la esperanza a la frustración.

La gran diversidad de demandas integradas en la indignación y la escasa afiliación previa a colectivos de trabajo político o social (Cammaerts y Van Audenhove, 2005) dificultaron la construcción de consensos reclamada por el discurso habermasiano que configura una identidad colectiva (Ortiz-Galindo, 2016a) necesaria para que la acción del movimiento sea diversificada y duradera (Larraña, 1999). 


\section{La construcción colectiva en el panorama posconvencional: la Plataforma de Afectados por la Hipoteca}

El análisis de las estructuras de movilización de la Plataforma de Afectados por la Hipoteca (PAH), en España, marca un punto de quiebre frente al tipo de acciones de protesta hasta ahora analizadas. Desde el punto de vista posconvencional, la PAH se sitúa en una lucha colectiva por el ejercicio de un derecho humano - la vivienda- (Ortiz-Galindo, 2016a). Sin dejar de actuar sobre un marco legal y normativo, se posiciona desde la búsqueda del ejercicio del derecho y realiza acciones diversas y constantes que, de forma coherente con el derecho y con una visión de justicia ligada a valores morales (Habermas, 1981; Alútiz, 2010; Martínez, 2011), intentan garantizar el ejercicio del derecho a individuos o colectivos privados de este.

\section{La estrategia}

La PAH genera constantes desafíos al sistema político en los que se desarrolla, pues su posicionamiento moral —un objetivo de carácter público (Ortiz-Galindo, 2016a) en principio superior a la norma social—impide, retrasa o simplemente cuestiona el ejercicio de la fuerza o la ejecución de la sanción normativa — convencional — sobre las acciones del movimiento (Giraldo-Luque, 2015). El análisis estratégico del sistema político, de sus vacíos y lagunas encontradas en el universo convencional, obliga a las instituciones a actuar de forma diferente y a buscar la integración de los desafíos en sus marcos normativos. Existe, por tanto, una personalización y adaptación de diversas acciones que proponen desafíos al sistema político (Anduiza, Cristancho y Sabucedo, 2014).

La estrategia de la PAH está definida por la construcción de un consenso previo - un escenario discursivo antecesor a la acción (Habermas, 2008) - alimentado por vínculos racionales decididos colectivamente y vinculados a una identidad (Touraine, 2005; Chomsky, 2011).

El marco racional que define al actor supera la perspectiva emotiva del contagio, pero integra esa lógica emocional (Bond et al., 2012) para alcanzar, bajo la mediatización de doble vía (Feenstra et al., 2016), mayor le- 
gitimidad y solidaridad hacia sus acciones. Las redes sociales desempeñan así un papel estratégico respecto de las convocatorias y la comunicación de sus acciones. A pesar de ello, la red social no se convierte en el movimiento y, por tanto, la expectación sobre ella es baja (Anduiza, Cristancho y Sabucedo, 2014). Existe una unidad discursiva sobre la acción realizada (Lynch, Freelon y Aday, 2017).

\section{Las acciones}

La intencionalidad de las acciones de la PAH (Castells, 1997) está definida hacia la satisfacción de derechos y no espera una respuesta institucional, adoptando, por tanto, las propuestas de Bentivegna (2006) y Ortiz-Galindo (2016a). Su marco y repertorio de acciones, consensuadas racionalmente (Habermas, 2008), se traducen en hechos que acercan el ejercicio del derecho y transforman la vida de las personas: en ocho años la PAH ha impedido más de 2045 desahucios y ha realojado a 2500 personas. Las acciones autónomas que garantizan derechos a personas que antes no podían ejercerlos generan desafíos sistémicos importantes a los centros de decisión pública. En el caso de la PAH, el movimiento provocó impulsos jurídicos y legislativos que integraban algunas de las demandas iniciales de la movilización (Carmona, 2017), que han transformado el sistema de hipotecas en España y recuperado una serie de derechos para los hipotecados. De esta forma, desmarcados de la institucionalidad, alcanzaron una mayor atención institucional al salirse de las prácticas de demanda convencionales.

En el marco del escenario posconvencional, los medios sociales se asumen como un factor de presión que acompaña las acciones realizadas. Son así una acción más que se vincula al intento por generar mayor solidaridad (Castells, 2012). El efecto contagio ante la defensa de un derecho y la transformación de las condiciones de vida de las personas actúa eficazmente en los diferentes medios de comunicación y redes sociales alternativas (Alonso-Muñoz y Casero-Ripollés, 2016).

\section{Los objetivos}

Las movilizaciones posconvencionales destacan por el recorrido histórico que les permite alcanzar consensos en sus estrategias, acciones y objetivos a largo plazo. La PAH, activa desde febrero de 2009, se traza unos objeti- 
vos consensuados (Chomsky, 2011) a partir del trabajo previo (Cammaerts y Van Adenhove, 2005) y que se convierten en la garantía de la identidad compartida y aceptada (Touraine, 2005).

El marco histórico ha permitido a la PAH construir, desde un espacio presencial y directo (Keane y Feenstra, 2014, p. 4), diversas líneas de acción —una de las cuales es la acción mediática - con una continuidad temporal que permite el cumplimiento de metas concretas sobre la garantía de los derechos fundamentales (Larraña, 1999).

La PAH destaca como movilización porque, dentro de sus acciones, encuadra un modelo de repetición sistemática para que sus miembros o grupos generen procesos autónomos de organización de la sociedad civil (Giraldo-Luque, 2015).

\section{Discusión}

El análisis de las movilizaciones plantea diálogos con algunas de las categorías planteadas por diferentes autores. Respecto de la estrategia, aunque el $15 \mathrm{M}$ y la Revolución egipcia aprovechan el contagio emotivo de la protesta (Bond et al., 2012; Coviello et al., 2014; Jones et al., 2017), las esperanzas se transforman rápidamente en miedo — se inaugura así una crítica al planteamiento de Castells (2012) al caracterizar el poder de las redes de indignación y esperanza - a partir de una respuesta institucional concreta, bien de tipo preconvencional, bien de tipo convencional (Alútiz, 2004).

Las acciones enmarcadas en un punto de mira institucional revelan que no hay pérdida de centralidad en las acciones de Egipto y de los indignados en España. En el caso de la PAH, es evidente que la idea principal del movimiento es garantizar, de forma autónoma, un derecho humano insatisfecho y, por tanto, asume las propuestas teóricas de Bentivegna (2006), Anduiza, Cristancho y Sabucedo (2014) y Ortiz-Galindo (2016a) de desvincular su acción del universo institucional. De igual forma, las metas propuestas por cada una de las movilizaciones hacen variar el tipo de respuesta - preconvencional, convencional o posconvencional - que el sistema político oferta cuando recibe una amenaza. Mientras en los dos primeros ca- 
sos se refuerza la legitimidad institucional al mantener el estado de cosas tradicional, en el último el que recibe solidaridad y altas dosis de legitimidad - a partir de una orientación a los principios de la justicia (Habermas, 1981) - es el movimiento social al alcanzar la satisfacción de un derecho y la readaptación sistémica, incluso normativa, de la demanda social (Carmona, 2017).

Si se analizan los objetivos, los casos de Egipto y el 15M no tienen continuidad temporal, una condición que para Larraña (2009) resulta inherente a un movimiento social. Una causa de lo anterior es, sin duda, la ausencia de construcción previa de consensos o de una identidad compartida para el establecimiento de una agenda específica de trabajo, una situación que se sitúa a distancia de lo expuesto por Cammaerts y Van Audenhove (2005). Si se analiza el caso posconvencional, el marco de asociación previo es el que va a determinar la unidad del grupo y la adquisición tanto de un consenso sobre sus diversas acciones (Habermas, 2005) como de una identidad colectiva (Touraine, 2005). Resaltar que, en la $\mathrm{PAH}$, es la discusión racional y no la emocional la que genera la cohesión y garantiza la longevidad de la organización social como movimiento (Giraldo-Luque, 2015).

En última instancia, al estudiar el papel que desempeñan las redes sociales y la comunicación en la movilización social, es necesario retomar a Lynch, Freelon y Aday (2017) cuando indican que el discurso mediático polarizó la protesta y alejó el sentimiento de unidad en el caso egipcio. En el $15 \mathrm{M}$, la centralidad dada a la comunicación convirtió a las redes sociales en el movimiento $y$, a pesar de que lograron acercarse a reconducir una autonomía mediática bajo la mediatización de doble vía (Feenstra et al., 2016), una parte fundamental de sus miembros fueron integrados, política y comunicativamente, al sistema institucional de óptica convencional (Casero-Ripollés et al., 2016). La PAH, por su parte, vincula su estrategia de comunicación como una acción integrada para el logro de sus objetivos de largo plazo y utiliza las redes para aumentar el rango de solidaridad social de sus logros. En este caso, los medios sociales son una vitrina alternativa a la dinámica mediática tradicional que les permite, efectivamente, 
salir del marco institucional y posicionar un discurso con otras lógicas de agenda (Feenstra et al., 2016; Ortiz-Galindo, 2016a). En cualquier caso, esa descentralización, en los movimientos pre-y convencionales, solo se ejerce desde el punto de vista mediático, pero no en la práctica de la acción política.

\section{Conclusión}

La clasificación de las expresiones de protesta de acuerdo con la perspectiva de los estadios del desarrollo moral aplicada al entendimiento de la realidad social implica la toma de posición sobre lo que significa constituir un movimiento social en el siglo XXI. Los casos estudiados, de acuerdo con el análisis propuesto, demuestran que la aparición como trending topic de una protesta o acción colectiva no determina su capacidad para incidir en la mejora de los derechos ciudadanos.

El grado de incidencia de una movilización está determinado por la estrategia que se plantee, el tipo de acción que ejecute y la clase de objetivo que persiga. 3500 asesinados demuestran que Egipto debe reinventarse para generar espacios democráticos en un sistema político que aplica la coacción en contra de expresiones de inconformidad.

Al mismo tiempo, los pocos cambios alcanzados por plataformas como el 15M demuestran la contradicción entre las redes sociales y los intereses de la movilización (Fuchs, 2014). Existe una tensión en el uso de la red como canal de protesta social y en su concepción como esfera pública. Los medios sociales juegan en favor de la convencionalidad sistémica dentro de la cual obtienen beneficios. De la indignación convertida en esperanza, el 15M degeneró en frustración.

En la orilla posconvencional, el movimiento social emerge como una construcción colectiva que sitúa su objetivo a largo plazo y orientado a la conquista de derechos. La construcción del consenso sobre las acciones guiado por la perspectiva moral de la justicia - permite trazar una estrategia que genera desafíos políticos y, al mismo tiempo, propone acciones concretas asumidas desde la autonomía. El uso de los medios de comunicación, incluidas las redes sociales, es una herramienta al servicio de la estra- 
tegia, pero no puede constituir la estrategia en sí misma. Es una definición que va de la mano con la propuesta de Ortiz-Galindo (2016a, pp. 167-169), para quien un cibermovimiento social implica la acción colectiva, se orienta al cambio social, persigue objetivos de carácter público, está motivado por un conflicto, tiene una identidad, mantiene una intencionalidad, presenta una continuidad temporal, se estructura reticularmente y se desarrolla en el ámbito de la sociedad civil, no de las instituciones.

\section{Referencias}

Alonso-Muñoz, L. y Casero-Ripollés, A. (2016). La influencia del discurso sobre cambio social en la agenda de los medios: el caso de la Plataforma de Afectados por la Hipoteca. OBETS: Revista de Ciencias Sociales, 11(1), 25-51. doi:10.14198/OBETS2016.11.1.02

Alútiz, J. C. (2004). Los estadios morales de la evolución social. Papers: Revista de Sociología, 74, 11-44.

Alútiz, J. C. (2010). Una introducción a la moral posconvencional desde la sociología y la filosofía política. Postconvencionales, 1, 8-19.

Amnesty International (2015). Generation Jail: Egypt's Youth go from Protest to Prison. Londres: Amnesty International.

Anduiza, E., Cristancho, C. y Sabucedo, J. M. (2014). Mobilization through online social networks: The political protest of the indignados in Spain. Information, Communication \& Society, 17(6), 750-764.

Bennett, W. L. y Segerberg, A. (2012). The logic of connective action: Digital media and the personalization of contentious politics. Information, Communication \& Society, 15(5), 739-768.

Bentivegna, S. (2006). Rethinking politics in the worlds of ICT. European Journal of Communication, 21(3), 331-343. 
Bond, R. M., Fariss, C. J., Jones, J. J., Kramer, A. D., Marlow, C., Settle, J. E. y Fowler, J. H. (2012). A 61-million-person experiment in social influence and political mobilization. Nature, 489(7415), 295-298. doi:10.1038/nature11421

Cammaerts, B. y Audenhove, L. van (2005). Online political debate, unbounded citizenship and the problematic nature of a transnational public sphere. Political Communication, 22(2), 179-196.

Camps, C. y Vergés, N. (2015). De la superación del miedo a protestar al miedo como estrategia represiva del 15M. Athenea Digital, 15(4), 129-154.

Carmona, A. M. (2017). La construcción por el Tribunal de Justicia de la Unión Europea de un stándard común de protección de derechos del consumidor en los procedimientos de ejecución hipotecaria. Teoría y Realidad Constitucional, 39, 307-332.

Casero-Ripollés, A. y Feenstra, R. A. (2012). The 15-M Movement and the new media: A case study of how new themes were introduced into Spanish political discourse. Media International Australia, 144(1), 68-76.

Casero-Ripollés, A., Feenstra, R. A. y Tormey, S. (2016). Old and new media logics in an electoral campaign: The case of Podemos and the two-way street mediatization of politics. The International Journal of Press/Politics, 21(3), 378-397.

Castells, M. (1997). La era de la información: economía, sociedad y cultura (vol. 2). México: Siglo XXI.

Castells, M. (2012). Redes de indignación y esperanza. Madrid: Alianza.

Ceron, A. y Memoli, V. (2016). Flames and debates: Do social media affect satisfaction with democracy? Social Indicators Research, 126(1), 225-240. https://doi.org/10.1007/s1 1205-015-0893-x 
Coviello, L., Sohn, Y., Kramer, A. D., Marlow, C., Franceschetti, M., Christakis, N. A. y Fowler, J. H. (2014). Detecting emotional contagion in massive social networks. PloS one, 9(3), e90315.

Chadwick, A. (2006). Internet politics. Nueva York: Oxford University Press.

Chomsky, N. (2011). Ocupemos el futuro. En G. Muñoz Ramírez (comp.), Indignados. México: Ediciones Bola de Cristal.

Dijck, J. van (2011). Facebook as a tool for producing sociality and connectivity. Television \& New Media, 13(2), 1-17.

Eltantawy, N. y Wiest, J. B. (2011). The Arab spring. Social media in the Egyptian revolution: Reconsidering resource mobilization theory. International Journal of Communication, 5, 1207-1224.

Feenstra, R. A., Tormey, S., Casero-Ripollés, A. y Keane, J. (2016). La reconfiguración de la democracia: el laboratorio político español. Granada: Comares.

Fuchs, C. (2014). Social media: A critical introduction. Los Ángees: Sage.

Giraldo-Luque, S. (2012). Modelo de análisis del uso de internet para promover la participación ciudadana en los procesos de toma de decisión pública (poderes ejecutivo y legislativo). Signo y Pensamiento, 31(61), 18-36.

Giraldo-Luque, S. (2015). Més enllà de Twitter: de l'expressió indignada a l'acció política. Vic: Eumo.

Habermas, J. (1981). La reconstrucción del materialismo histórico. Madrid: Taurus.

Habermas, J. (2005). Tres modelos de democracia: sobre el concepto de democracia política deliberativa. Polis: Revista de la Universidad Bolivariana, 4(10). 
Habermas, J. (2008). Comunicación política en la sociedad mediática: el impacto de la teoría normativa en la investigación empírica. Revista Libero, 11(21), 9-22.

Human Right Watch (2014). All According to Plan The Rab'a Massacre and Mass Killings of Protesters in Egypt. Nueva York: Human Rights Watch.

Jones, J. J., Bond, R. M., Bakshy, E., Eckles, D. y Fowler, J. (2017). Social influence and political mobilization: Further evidence from a randomized experiment in the 2012 U.S. presidential election. PLoS ONE, 12(4), e0173851. https://doi.org/10.1371/journal. pone. 0173851

Keane, J. y Feenstra, R. A. (2014). Democracia monitorizada en España: nuevas formas de participación política en el marco de la era digital. Telos: Cuadernos de Comunicación, 98, 48-57.

Kolhberg, L. (1992). Psicología del desarrollo moral. Bilbao: Desclée de Brouwer.

Kramer, A. D., Guillory, J. E. y Hancock, J. T. (2014). Experimental evidence of massive-scale emotional contagion through social networks. Proceedings of the National Academy of Sciences, 111(24), 8788-8790. doi: $10.1073 /$ pnas.1320040111

Larraña, E. (1999). La construcción de los movimientos sociales. Madrid: Alianza.

Lévy, P. (2002). Ciberdemocracia: ensayo sobre filosofía politica. Barcelona: UOC.

Lynch, M., Freelon, D. y Aday, S. (2017). Online clustering, fear and uncertainty in Egypt's transition. Democratization, 24(6), 1159-1177. http://dx.doi.org/10.1080/13510347.2017.1289179

Martínez, D. (2011). Reformulación de la teoría del desarrollo moral de Kohlberg realizada pr Habermas. Persona y Sociedad, 25(2), 11-35. 
McLuhan, M. (1969). La galaxia Gutemberg: génesis del "homo typographicus". Madrid: Aguilar.

Micó, J. L. y Casero-Ripollés, A. (2014). Political activism online: Organization and media relations in the case of $15 \mathrm{M}$ in Spain. Information, Communication \& Society, 17(7), 858-871. DOI: http://dx.doi.or g/10.1080/1369118X.2013.830634

Naber, N. y Said, A. (2016). The cry for human rights: Violence, transition, and the Egyptian revolution. Humanity: An International Journal of Human Rights, Humanitarianism, and Development, 7(1), 71-90. DOI: 10.1353/hum.2016.0008

Natale, S. y Ballatore, A. (2014). The web will kill them all: New media, digital utopia, and political struggle in the Italian 5-Star Movement. Media, Culture \& Society, 36(1), 105-121. DOI: 10.1177/0163443713511902

Ortiz-Galindo, R. (2016a). Los cibermovimientos sociales: una revisión del concepto y marco teórico. Communication \& Society, 29(4), 165-183.

Ortiz-Galindo, R. (2016b). Estrategias de comunicación interpersonal y pública en los movimientos sociales: transformaciones de las redes informales y de los repertorios en la era de la web social. OBETS: Revista de Ciencias Sociales, 11(1), 211-254. DOI: 10.14198/ OBETS2016.11.1.09

Piaget, J. (1977). El criterio moral del niño. Barcelona: Fontanella.

Salzman, R. (2016). Exploring social media use and protest participation in Latin America. Journal of Latin American Communication Research, 5(2), 72-85.

Sunstein, C. R. (2017). \#Republic: Divided democracy in the age of social media. Princeton, NJ: Princeton University Press. 
Torrego, A. y Gutiérrez, A. (2016). Ver y tuitear: reacciones de los jóvenes ante la representación mediática de la resistencia. Comunicar, 47, 9-17. https://doi.org/10.3916/C47-2016-01

Touraine, A. (2005). Un nuevo paradigma: para comprender el mundo de hoy. Barcelona: Paidós.

Tufte, T. (2015). Comunicación para el cambio social. Barcelona: Icaria.

Wiener, N. (1969). Cibernética y sociedad. Buenos Aires: Suramericana.

Žižek, S. (2010). El acoso de las fantasías. Madrid: Siglo XXI. 